ARCHIVO ESPAÑOL DE ARTE, LXXXIV, 334

ABRIL-JUNIO 2011, pp. 171-176

ISSN: 0004-0428

\title{
UNA NUEVA PINTURA INÉDITA DE ALONSO DEL ARCO. ESTUDIO ICONOGRÁFICO Y DOCUMENTAL
}

\begin{abstract}
El presente artículo da a conocer y estudia un nuevo trabajo inédito del fecundo pintor madrileño de la segunda mitad del siglo XVII, Alonso del Arco, añadiendo así una nueva obra a su ya extenso catálogo.

Palabras Clave: Alonso del Arco; Escuela Madrileña; Siglo XVII; Pintura.

\section{A NEW UNPUBLISHED PAINTING BY ALONSO DEL ARCO: AN ICONOGRAPHIC AND DOCUMENTARY STUDY}

This article concerns an unpublished painting by Alonso del Arco, a prolific artist of the Madrid School during the second half of the $17^{\text {th }}$ century, thereby adding a new work to his already extensive catalogue.

Key words: Alonso del Arco; Madrid School; $17^{\text {th }}$ century; Painting.

Uno de los más prolíficos autores de la escuela pictórica madrileña de la segunda mitad del siglo XVII, es sin duda Alonso del $\mathrm{Arco}^{1}$, pero pese a lo abundante de su obra ${ }^{2}$, hacía bastante tiempo que no se daba a conocer ningún cuadro suyo nuevo ${ }^{3}$.

Pionera en un primer (y hasta ahora único) intento de catalogación sistemática de sus obras, fue Natividad Galindo San Miguel $^{4}$, que ha continuado después ocupándose del pintor, dando a conocer algunas obras ${ }^{5}$, estudiando otras pertenecientes a museos nacionales ${ }^{6}$ e in-

\footnotetext{
${ }^{1}$ Se trata de uno de los pintores de "segunda fila" o "menores" del barroco madrileño, así definidos por destacados investigadores. Véase al respecto URREA FERNÁNDEZ, Jesús, “Obras de pintores menores madrileños: B. de Castrejón, A. Van de Pere y P. Ruiz González", Boletín del Seminario de Estudios de Arte y Arqueología (BSAA), 1975, tomos 40-41, pp. 707-712; Id., "José García Hidalgo y Diego González de Vega: más obras de pintores menores madrileños", Boletín del Seminario de Estudios de Arte y Arqueología (BSAA), 1976, tomo 42, pp. 489-494; Id., "Alonso del Arco y José García Hidalgo: más obras de pintores menores madrileños (II)", Boletín del Seminario de Estudios de arte y Arqueología (BSAA), 1979, tomo 45, pp. 482-485; RIVERA, Javier, "Francisco de Lizona y Alonso del Arco: dos pinturas de maestros menores madrileños", Boletín del Seminario de Estudios de Arte y Arqueología (BSAA), tomo 47, 1981, pp. 477-480. Alonso del Arco desarrolló su actividad pictórica durante el reinado de Carlos II. Nació en Madrid en 1635 y una minusvalía física (era sordo de nacimiento) no le impidió aprender su oficio de la mano de su maestro Antonio de Pereda, hechos que le valieron el sobrenombre de "sordillo de pereda". Destacó como retratista y pintor de pintura religiosa. Para su biografía es de referencia la obra de Antonio Palomino, El Museo Pictórico y Escala Óptica. Tomo III. El parnaso español pintoresco y laureado, Madrid, 1715-1724, ed. Aguilar, 1947, reimpr. 1988, pp. 477-480.

${ }^{2}$ Ya Palomino señalaba su extraordinaria fecundidad: "Y finalmente pintó tanto, que apenas hay iglesia, o casa en esta Corte, donde no haya algo suyo; y asimismo en los lugares del contorno, hasta en la ciudad de Toledo, donde he visto muchas cosas suyas"; Ibid., p. 479.

3 Recientemente se han subastado varios lienzos suyos. Una "Muerte de Cristo" en Alcalá Subastas, mayo de 2010, lote 424 y "El taller de Nazaret" en Ansorena, subasta 313, lote 323 (firmado).

4 Galindo San Miguel, Natividad, "Alonso del Arco", Archivo Español de Arte, n. ${ }^{\circ} 180,1972$, pp. 347-386.

5 Galindo San Miguel, Natividad, "Dos nuevos cuadros de Alonso del Arco", Archivo Español de Arte, n. ${ }^{\circ}$ 183, 1973, p. 353; Id., "Un cuadro de Alonso del Arco en el ayuntamiento de Valencia", Archivo Español de Arte, n. ${ }^{\circ} 215$, 1981, p. 363.

${ }^{6}$ Galindo San Miguel, Natividad, "Presencia de Alonso del Arco en los fondos del Museo del Prado", Boletín del Museo del Prado, Vol. 4, n. ${ }^{\circ}$ 11, 1983, pp. 111-114.
} 
cluso ofreciéndonos su faceta de fresquista ${ }^{7}$, algo importante si tenemos en cuanta la escasez de pintores españoles dedicados a esta técnica. Con anterioridad ya se había ocupado de sacar a la luz algunas obras el profesor Valdivieso ${ }^{8}$. A esto cabe sumar los numerosos artículos que periódicamente han ido apareciendo a lo largo de los años en los que se mostraban nuevas obras del pintor $^{9}$. Sin embargo, a pesar de este rico panorama bibliográfico y de la abundancia de obra conservada, no se ha realizado un catálogo actualizado. Sería deseable pues, la puesta al día y revisión de lo conocido, con alguna monografía actualizada sobre tan fecundo artista.

Recientemente hemos tenido la ocasión de localizar un nuevo trabajo suyo, hasta ahora inédito y de importancia, si tenemos en cuenta la calidad general de la obra, la iconografía representada y la perfecta datación y documentación del lienzo.

Se trata de una Aparición de la Virgen y el niño a San Felipe Neri (fig. 1), firmado y fechado en el ángulo inferior izquierdo: Alonso del Arco.f. 1698 (fig. 2) ${ }^{10}$. El lienzo representa una escena clásica relacionada con el santo florentino ${ }^{11}$. En primer plano observamos al santo sacerdote que, arrodillado y arrobado, contempla la aparición divina de la virgen María con el niño en brazos. San Felipe, vestido con sotana y ferraiolo, aparece rodeado de los elementos iconográficos tradicionales con que se le suele representar: en primer lugar se puede ver el que es considerado su atributo personal, un corazón en llamas en su pecho, emblema de su Congregación. En el pavimento aparecen el capelo cardenalicio y la mitra, que representan las dignidades que el Papa le ofreció y a las que renunción ${ }^{12}$. Las azucenas, símbolo de los santos no mártires que se distinguieron por su pureza y castidad, completan el conjunto iconográfi-

\footnotetext{
7 Galindo San Miguel, Natividad, "Alonso del Arco, un fresquista inédito”, Boletín del Seminario de Estudios de Arte y Arqueología (BSAA), Tomo 46, 1980, pp. 451-460.

8 VAldivieso GonzÁlez, Enrique, "Tres pinturas de Alonso del Arco", Boletín del Seminario de Estudios de Arte y Arqueología: BSAA, Tomo 36, 1970, pp. 521-524; Id., "Tres nuevas obras de Alonso del Arco", Boletín del Seminario de Estudios de Arte y Arqueología: BSAA, Tomo 38, 1972, pp. 534-538.

9 Aparte de los artículos ya citados, véase: Ramallo AsEnSIO, Germán Antonio, "Dos Inmaculadas desconocidas, de Palomino y Alonso del Arco, en la Clausura del Convento del Sacramento", Miscelánea de arte, 1982, pp. 183-187; Martínez Ripoll, Antonio, "Una pintura inédita de Alonso Arco en Murcia", Archivo Español de Arte, n. ${ }^{\circ}$ 223, 1983, pp. 289-291; Seguí GonzÁlez, Mónica, "Restitución a Alonso del Arco de un cuadro atribuido a Alonso Cano", Archivo Español de Arte, n. ${ }^{\circ} 223,1983$, p. 297; GonZÁlez SANTos, Javier, "Obras de Alonso del Arco en Asturias", Liño: Revista anual de historia del arte, n. ${ }^{\circ}$ 5, 1985, pp. 227-234; ARNAIz, José Manuel, "Dos cuadros inéditos de Alonso del Arco y una puntualización", Archivo Español de Arte, n. ${ }^{\circ}$ 233, 1986, pp. 90-93; MÜLlER, Priscila E., "La Virgen del Popolo, por Alonso del Arco y una de María Villamor", Archivo Español de Arte, n. ${ }^{\circ}$ 241, 1988, pp. 71-72; NicOLAU CAStro, Juan, "Tres nuevos lienzos de Alonso del Arco en Toledo", Archivo Español de Arte, n. ${ }^{\circ}$ 255, 1991, pp. 372374; Capel Margarito, Manuel, "Otros cuadros y otro inédito de Alonso del Arco, en la Casa-Museo de los Pisa, de Granada", Archivo Español de Arte, n. ${ }^{\circ}$ 265, 1994, pp. 91-94; Rivera DE las Heras, José Ángel, "Dos obras de Carreño de Miranda y Alonso del Arco en Zamora", Boletín del Seminario de Estudios de Arte y Arqueología (BSAA), Tomo 64, 1998, pp. 395-404; DelEndA, Odile, "Dos lienzos firmados por Alonso del Arco en la parisina iglesia Saint-François de Sales", Archivo Español de Arte, n. ${ }^{\circ} 307,2004$, pp. 301-304.

${ }^{10}$ Madrid, colección particular. Óleo sobre lienzo, $2,11 \times 1,47 \mathrm{~cm}$.

${ }^{11}$ Felipe Neri, el llamado Apóstol de Roma, nació en Florencia en 1515. Siendo joven marchó a Roma donde conoció a Ignacio de Loyola y donde se ordenó sacerdote. En la Ciudad Eterna fundó un pequeño oratorio que sería reconocido por Gregorio XIII como Congregación. Su obra tendrá una particularidad: predecesor de tiempos más modernos, consiguió que los sacerdotes del oratorio, seculares, vivieran en comunidad sin realizar votos. Falleció en 1595 después de haber renunciado al episcopado y al cardenalato que se le había ofrecido años antes por el papa Sixto V. Para acercarse a la vida de este santo italiano, pueden consultarse las siguientes obras: BeLLido, Juan Félix, La conquista de la libertad: vida de San Felipe Neri, Bilbao, Desclée de Brouwer, 1998; CAPELATro, Alfonso, Vida de san Felipe Neri, Barcelona, establecimiento tipográfico "La hormiga de Oro", 1895; o CistellinI, Antonio, San Felipe Neri: breve historia de una vida grande, Alcalá de Henares, Madrid, A. Alba Alarcos, 2009.

12 RoIG, Juan Fernando, Iconografía de los Santos, Barcelona, ediciones Omega, 1991, p. 108.
} 


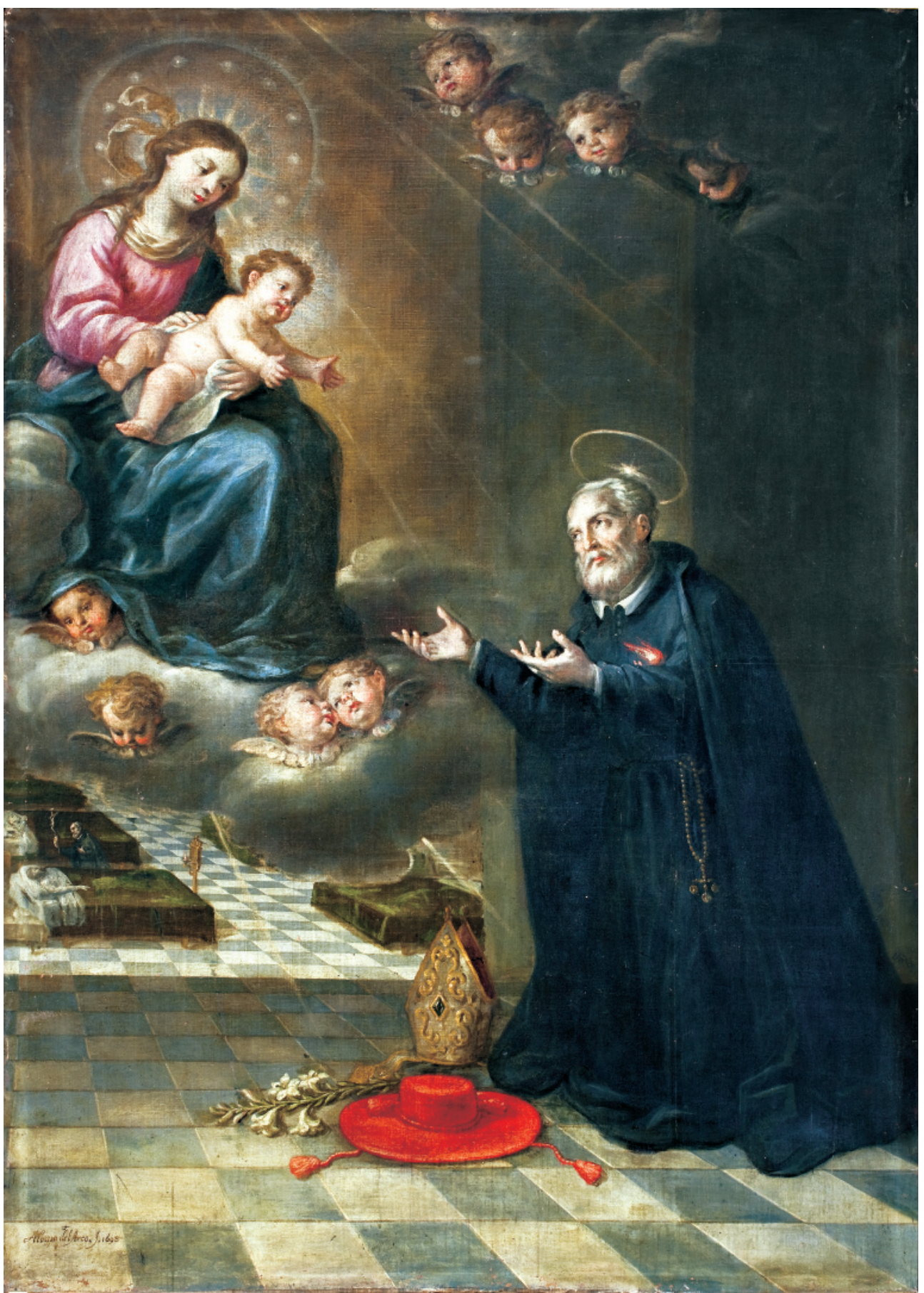

Fig. 1. La aparición de la Virgen y el niño a San Felipe Neri, Alonso del Arco, Madrid, Colección particular.

Arch. esp. arte, LXXXIV, 334, ABRIL-JUNIO 2011, 171-176, ISSN: 0004-0428 


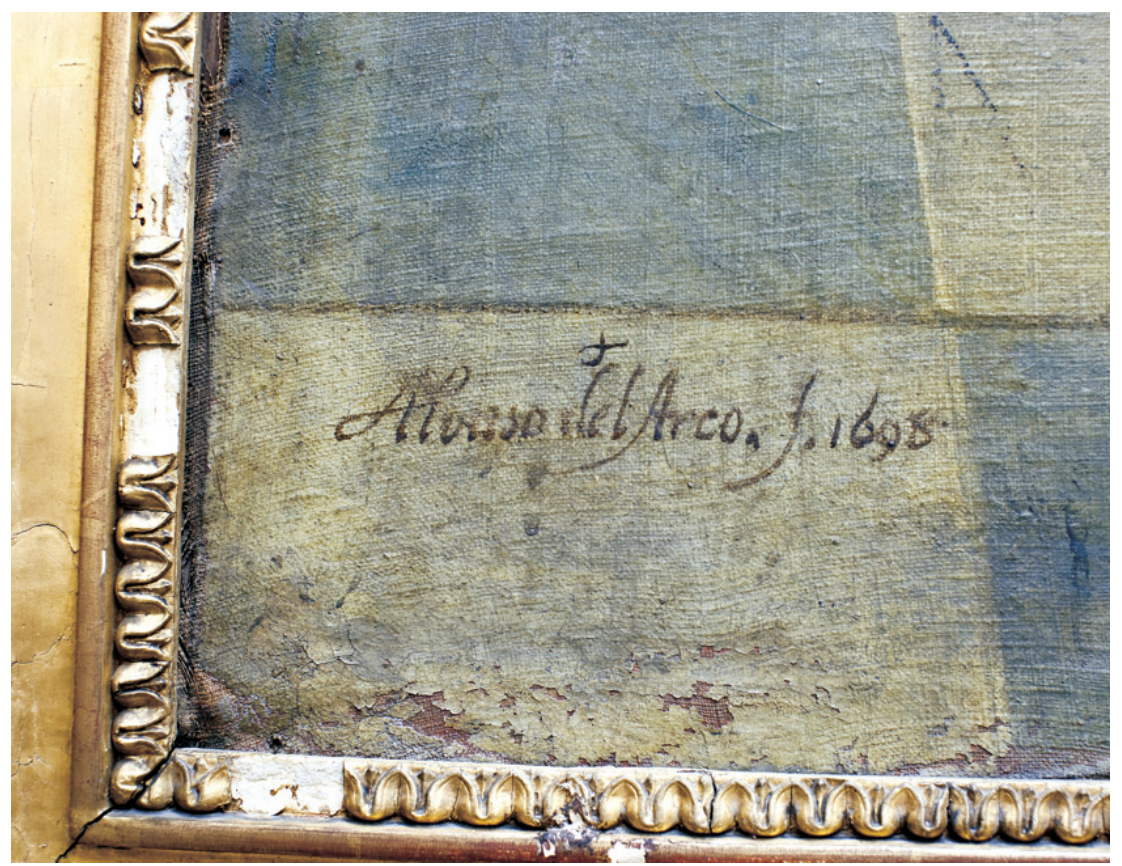

Fig. 2. Detalle de la Firma.

$\mathrm{co}^{13}$. En la parte superior, sobre un trono de nubes, la Virgen sostiene sobre su regazo a un inquieto niño Jesús que extiende sus brazos hacia el santo. Bajo ellos y arriba a la derecha varias cabezas de querubines contemplan la aparición. Al fondo del lienzo observamos otra escena desarrollada en una estancia aneja, que refleja muy gráficamente la labor asistencial a enfermos, llevada a cabo por el mismo San Felipe, que era el leiv motiv existencial de su Congregación.

A pesar de estar tremendamente oscurecido debido a la gruesa capa de suciedad acumulada, muy destensado y hasta desprendido del bastidor, lo que ha provocado algunos desgarros en las zonas de los clavos y desprendimientos de materia pictórica, su estado de conservación es considerablemente bueno teniendo en cuenta además las condiciones ambientales de su ubicación, expuesto a constantes cambios bruscos de temperatura y humedad. La superficie pictórica se conserva prácticamente intacta, salvo algunos desprendimientos, sobre todo en los ángulos, y se halla en su estado prístino pues no se aprecian repintes modernos. Es también patente el gran añadido de un trozo de lienzo rectangular en la mitad derecha. Añadido original para alcanzar las medidas requeridas del cuadro, sin que se deba a restauración alguna o reentelado posterior. La parte trasera del lienzo no presenta ninguna inscripción ni, como decimos, reentelado, siendo el gran "parche" original.

La obra pertenece a los últimos años de actividad del pintor, y son visibles algunas de las características técnicas y formales del mismo, sobre todo en la pincelada deshecha y en los peculiares rasgos fisonómicos del tipo iconográfico y humano de la Virgen, del que contamos con numerosísimos testimonios. Del mismo modo, la figura del Santo se asemeja mucho a la de San Antonio Abad del mercado anticuario madrileño, con ese volumen tan característico de sus

13 Ibid. p. 276. 
personajes y los marcados pliegues de los ropajes.

El cuadro es correcto y de calidad elevada sobre todo en lo que a las figuras principales se refiere y de modo particular en el rostro y los ropajes de la Virgen, así como el rostro y las manos de San Felipe. La participación de taller, tan usual en los lienzos de Alonso del Arco, parece advertirse aquí en la escena secundaria desarrollada como fondo de la composición, ejecutada de modo mucho más ligero y sintético.

Resulta bastante patente que Alonso del Arco sigue fielmente modelos de su maestro Pereda, como hará en muchas otras ocasiones. Las similitudes compositivas de este lienzo con las dos versiones de San Francisco en la Porciúncula (Barcelona, Colección particular, y Valladolid, Museo Nacional de Escultura como depósito del Prado), saltan a la vista incluso en la colocación de los atributos de los respectivos santos (fig. 3) ${ }^{14}$.

En cuanto a la procedencia del cuadro, se trata con toda probabilidad de uno de los lienzos que menciona Palomino en el antiguo Hospital General, donde tenía su sede la Congregación de San Felipe Neri en Madrid, aunque desgraciadamente sin hacer

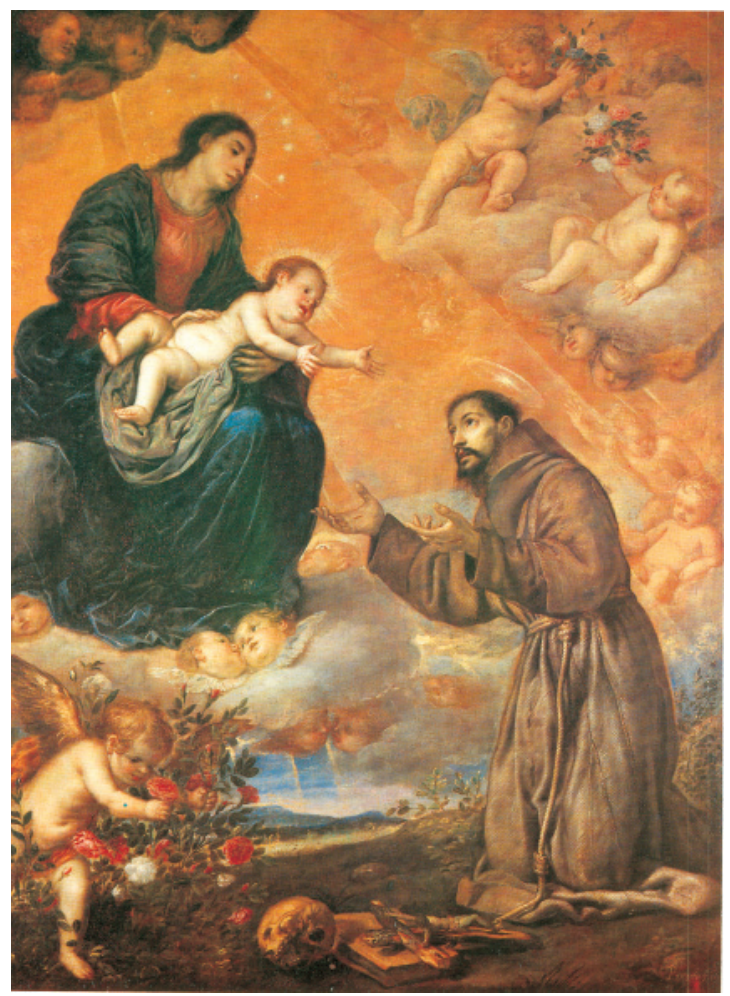

Fig. 3. San Francisco en la Porciúncula, Antonio de Pereda, Valladolid, Museo Nacional de Escultura, Depósito del Museo del Prado. alusión a los temas ${ }^{15}$. Hoy en día se conserva en una colección particular madrileña ${ }^{16}$ que además guarda un importantísimo archivo en el que hemos podido hallar algunas referencias casi contemporáneas al lienzo, que debió ser encargado por los filipenses para decorar la iglesia del Hospital, permaneciendo allí hasta su traslado. El cuadro aparece en dos inventarios del archivo. El primero, de 1794, habla de esta pintura refiriéndose a ella como "Otra Pintura de nuestro Santo Patriarca San Phe. Nery con Marco tallado y dorado de dos Varas y media de alto y dos de Ancho quese halla en la Sacrisitia"17. El segundo inventario no habla de su ubicación, pero dice que se trata de "Una pintu-

14 Pérez Sánchez, Alfonso E., D. Antonio de Pereda (1611-178) y la pintura madrileña de su tiempo, catálogo de la exposición, Madrid, 1978-1979, n. ${ }^{\text {os }} 23$ y 35; La pintura madrilena del secolo XVII, catálogo de la exposición, Roma, 1991-1992, n. ${ }^{\circ} 22$. El mismo tema de Alonso del Arco en el Convento de San Pascual de Madrid, resulta prácticamente un trasunto del de Valladolid; Galindo SAn Miguel, Natividad, "Alonso del Arco", op. cit., p. 373.

15 Palomino de Castro y Velasco, Antonio, El Museo Pictórico y escala óptica..., op. cit., p. 479.

${ }^{16}$ Hay también un par de Vírgenes del Popolo en muy mal estado de conservación, que parecen corresponder asimismo a la mano de Alonso del Arco.

17 Archivo Colección privada madrileña (ACPM), "Inventario de las Alaxas, Ornam.tos, muebles y demás Efectos pertenecientes a la Congreg.on de nro. P. Sn. Felipe Neri de Seglares siervos de los Pobres Enfermos del Hospital Gral. de esta Corte. Año de 1794" (sin foliar). 
ra del Sor. S. Felipe Nery con marco dorado de dos y media vs. de alto por dos de ancho"18. Un último documento suelto, atestigua que la obra no formó parte de la restauración de objetos y obras de arte de la capilla llevada a cabo por Gerardo Meléndez en 1901.

En la iglesia del convento de las Trinitarias Descalzas de Madrid se conserva también otra aparición a San Felipe Neri firmada por nuestro pintor ${ }^{19}$.

\author{
CARLOS Nieto SÁNCHeZ \\ $\mathrm{UCM}$ \\ y Álvaro Pascual CHENel \\ $\mathrm{IH}, \mathrm{CCHS}, \mathrm{CSIC}$
}

18 ACPM, "Ynventario general de las Alajas, Ropas y demás enseres que existen en el oratorio de la Congregación de N. Padre y Patriarca S. Felipe nery, Siervos de los Pobres enfermos del Hospital General de esta Corte." (1821) (sin foliar).

19 Galindo San Miguel, Natividad, "Alonso del Arco", op. cit., p. 359; Inventario Artístico de Madrid, tomo II, edificios religiosos de los siglos XVI-XVIII, p. 220.

Arch. esp. arte, LXXXIV, 334, ABRIL-JUNIO 2011, 171-176, ISSN: 0004-0428 